

Revista de lingüística, filología y traducción
PONTIFICIA UNIVERSIDAD CATÓLICA DE CHILE FACUITAD DE LETRAS

\title{
Capacidades lingüísticas shipibo-castellano en textos escritos por escolares bilingües de Ucayali (Perú) ${ }^{1}$
}

Shipibo-Spanish language skills in texts written by bilingual schoolchildren in Ucayali (Peru)

\section{Inés $\mathbf{M}^{\mathbf{a}}$ García-Azkoaga}

Universidad del País Vasco (UPV/EHU)

España

\section{Karina Sullón Acosta}

Universidad del País Vasco (UPV/EHU)

España

Número especial

Las lenguas amerindias en Iberoamérica: retos para el siglo XXI

2017
ONOMÁZEIN - Número especial

Las lenguas amerindias en Iberoamérica: retos para el siglo XXI (2017): 153-170 DOI: 10.7764/onomazein.amerindias.09

\section{(c) $\underset{B Y}{(i)} \bigodot_{\mathrm{ND}}$}

Inés $\mathbf{M}^{\mathbf{a}}$ García-Azkoaga: Departamento de Lengua Vasca y Comunicación, Universidad del País Vasco (UPV/ EHU), España. I Correo electrónico: ines.garciaazkoaga@ehu.eus

Karina Sullón Acosta: Universidad del País Vasco (UPV/EHU), España.

| Correo electrónico: karinasullon@gmail.com 


\section{Resumen}

La mayoría de estudios sobre el shipibo-konibo, lengua de la familia lingüística pano hablada principalmente en el departamento amazónico de Ucayali (Perú), se centra en el análisis gramatical. Son escasos los estudios sobre las capacidades lingüísticas y comunicativas en shipibo y castellano de niños y niñas bilingües escolarizados en el modelo de educación intercultural bilingüe (EIB). En este sentido, nuestra propuesta se destina al análisis de un género textual determinado, las recetas escritas en esas dos lenguas por niños y niñas de entre 11 y 13 años de edad que comparten el mismo espacio de aprendizaje. Siguiendo la metodología utilizada en investigaciones sobre adquisición y aprendizaje de lenguas en el contexto de una lengua minorizada (Idiazábal y García-Azkoaga, 2015; Manterola, 2012; Díaz de Gereñu y García-Azkoaga, 2016, entre otros), abordamos el estudio desde una perspectiva sociodiscursiva que considera el texto como la unidad comunicativa pertinente para dar cuenta de las capacidades de los alumnos. Analizamos los siguientes aspectos textuales: estructura, conexión, cohesión y marcas de responsabilidad enunciativa. Los resultados obtenidos nos permiten identificar los recursos empleados por los alumnos y conocer así los aspectos en los que debemos incidir para contribuir a mejorar la comunicación escrita del género textual analizado.

Palabras clave: educación bilingüe; lenguas minoritarias; aprendizaje de lenguas; género textual; shipibo-konibo.

\section{Abstract}

The majority of studies on the Shipibo-Konibo, language of the Pano linguistic family spoken mainly in the Amazonian department of Ucayali (Peru), are focused on its grammatical analysis. There exist few studies devoted to analyse the communicative skills in Shipibo and Spa-

1 Esta contribución se ha realizado gracias a proyectos de investigación financiados por el Gobierno vasco (IT676-13 e IT983-16-GIC15/129) y el MEC del Gobierno de España (FFI2012-37884-C03-01).

Nuestro más sincero agradecimiento a los docentes y alumnos de Puerto Firmeza por su ayuda y su buena disposición a la hora de recopilar el corpus. A la docente Ibeth Sánchez Rojas por su apoyo en la revisión de las recetas. Asimismo, nuestra gratitud a la Dirección General de Educación Básica Alternativa, Intercultural Bilingüe y de Servicios Educativos en el Ámbito Rural-DIGEIBIR del Ministerio de Educación del Perú por fomentar investigaciones que aporten al desarrollo de la ElB. 
nish of bilingual children enrolled in the intercultural bilingual education model (EIB). In this sense, we analyze a particular textual genre, recipes written in those languages by children between 11 and 13 who share the same learning environment. Following the methodology used in research on acquisition and language learning in the context of a minority language (Idiazábal and Garcia-Azkoaga, 2015; Manterola, 2012; Díaz de Gereñu and Garcia-Azkoaga, 2016, among others), we address the study from a socio-discursive perspective that considers the text as piece to account for the abilities of students. We analyze the following textual aspects: structure, connection, cohesion, and enunciative marks like modalization and voices. The results allow us to identify the resources used by the students and to distinguish the aspects on which we can influence to help them improve written communication of the analyzed text genre.

Keywords: bilingual education; minority languages; language learning; text genre; ShipiboKonibo. 


\section{Introducción}

El shipibo-konibo (en adelante shipibo) es una de las 47 lenguas originarias que coexisten actualmente en el Perú junto al castellano. Es una lengua de la familia lingüística pano y se habla en cinco departamentos del Perú: Ucayali, Loreto, Madre de Dios, Huánuco y Lima. Es una lengua aglutinante y ergativa que cuenta aproximadamente con 22.500 hablantes. La lengua shipibo está en proceso de estandarización. El alfabeto consensuado por la población fue oficializado en el 2007, mediante una resolución directoral emitida por el Ministerio de Educación del Perú, fecha desde la cual se han elaborado diferentes materiales escolares. Sin embargo, es a partir del 2012 cuando el Ministerio de Educación empieza elaborar los cuadernos de trabajo en shipibo para los niños que hablan esa lengua y que estudian en las instituciones de educación intercultural bilingüe (EIB), materiales que fueron usados por primera vez en 2013.

El shipibo se aprende en algunas comunidades como segunda lengua. Uno de los retos de las acciones promovidas dentro del marco de la educación intercultural bilingüe (EIB) es, precisamente, hacer llegar a esas comunidades hablantes de lenguas originarias minoritarias la posibilidad de acceder a una educación bilingüe que facilite la alfabetización y el desarroIlo lingüístico en la lengua originaria y en la lengua mayoritaria (castellano). Es una iniciativa importante si se tiene en cuenta, como señalan Amorrortu y otros (2006), que la educación es uno de los factores fundamentales para el desarrollo del multilingüismo, tal y como reconoce también la propia Organización de las Naciones Unidas en una resolución adoptada en su Asamblea General del 8 de junio de 2007.

A este respecto, téngase en cuenta que la EIB aborda la escolarización inicial, primaria y secundaria de los alumnos teniendo en cuenta cuatro escenarios para los alumnos: 1) tienen la lengua originaria como L1 y el castellano como L2; 2) usan aceptablemente ambos idiomas, algunos son bilingües de cuna; 3) hablan predominantemente el castellano, tienen un nivel básico de la lengua originaria, y 4) tienen como Lı el castellano y como L2 la lengua originaria. La institución educativa objeto de esta investigación se ubica en el primer escenario y los docentes bilingües shipibo-castellano utilizan ambas lenguas como lenguas vehiculares de la escuela. Son, además, esos mismos docentes los que deciden qué lengua utilizar en que área y en qué momento, atendiendo a las necesidades de sus alumnos, tanto al comienzo como a lo largo del curso.

El objetivo de este trabajo es acercarnos a las capacidades lingüístico-comunicativas que desarrollan los niños y niñas educados en el sistema bilingüe shipibo-castellano de la EIB, lo que implica considerar la situación de comunicación en la que se desarrolla la actividad verbal. No es lo mismo escribir o contar una narración que una receta, escribir un texto como tarea de escritura descontextualizada o escribir un texto situado que responde a un objetivo 
comunicativo concreto. Por ello, nos planteamos aquí la tarea de analizar las capacidades escritas de los niños bilingües shipibo-castellano a través de la escritura de recetas de cocina y de hacerlo por medio de una metodología que proporciona información sobre las capacidades comunicativas de los alumnos y también sobre su competencia gramatical y ortográfica. Esta investigación proporciona, asimismo, un diagnóstico de la situación que será de utilidad para una futura adaptación y optimización de las metodologías de enseñanza y los recursos didácticos existentes. Así, utilizaremos una metodología que explica el funcionamiento de la lengua a partir de los textos (Bronckart, 1996), orientación que ha sido utilizada también en diversos trabajos realizados sobre las capacidades de alumnos bilingües vasco-español que se citan más adelante.

En este contexto, la noción de capacidad lingüística se entiende como la habilidad de actuar verbalmente en situaciones comunicativas diversas; dicho de otra manera, la capacidad de producir un texto (oral o escrito) adaptado al contexto comunicativo, siendo el texto la unidad comunicativa que un individuo produce en el transcurso de la actividad verbal. Desde esta perspectiva, el texto (y, más concretamente, el género textual) es una unidad comunicativa, la unidad de trabajo de la didáctica de las lenguas (De Pietro y Schneuwly, 2003), pero es también una valiosa herramienta para dar cuenta de las capacidades lingüístico-comunicativas desarrolladas por los escolares en sistemas de educación bi/plurilingüe como muestran los trabajos de Idiazábal y García-Azkoaga (2015) sobre las recetas de cocina, o los de Manterola (2012), García-Azkoaga y otros (2009), García-Azkoaga e Idiazábal (2015), Díaz de Gereñu y García-Azkoaga (2016) sobre las narraciones, entre otros. En consecuencia, la evaluación de las capacidades lingüísticas debe hacerse por medio de metodologías que tengan en cuenta esa dimensión empírica y contextualizada del uso de la lengua, que ofrecen los géneros textuales (Dolz y Gagnon, 2010). Desde esta perspectiva, se analizan recetas de cocina escritas en shipibo y en castellano por niños de $5^{\circ}$ y $6^{\circ}$ grado de primaria de una escuela de EIB de Ucayali. Como señalan Idiazábal y García-Azkoaga (2015), la receta de cocina es un texto normalmente breve y con una estructura bastante sencilla que, además de tener un gran potencial didáctico, puede ser utilizado como herramienta para la evaluación de la competencia verbal.

Por otra parte, si bien existen investigaciones sobre el shipibo (Valenzuela, 2001, 2006, 2013; Zariquiey, 2006, etc.), no se han encontrado estudios centrados en la dimensión comunicativa de esta lengua ni trabajos previos sobre el desarrollo de las capacidades bilingües shipibo-castellano de alumnos de EIB, aspectos en los cuales se incide en este artículo.

\section{Metodología}

Desde el punto de vista de la comunicación, cuando un sujeto produce un texto, además de identificar o elegir el género textual más acorde con las exigencias generales de la situación 
comunicativa - en este caso la receta de cocina-, el emisor debe adaptarlo a las exigencias particulares del contexto de enunciación. En el caso de la receta, se trata de un texto que básicamente proporciona una serie de instrucciones para la elaboración de un plato de cocina. Así, puede ser considerado un texto procedimiental (Calvo, 2001) o, en terminología de Dolz, Noverraz y Schneuwly (2001), un género textual para regular comportamientos. En cualquier caso, una receta de cocina contiene un conjunto de indicaciones que, a partir de la combinación de unos ingredientes y de su manipulación o tratamiento, conduce a la preparación de una comida. Es un género de texto conocido y socialmente accesible de forma tanto oral como escrita para la gran mayoría de los hablantes. Según se avanzó anteriormente, para su análisis empleamos el esquema de la arquitectura textual de Bronckart (1996), en el que integramos las características subrayadas por Adam (2001) para la receta de cocina en lo referente a la estructura, a la organización temporal del proceso de elaboración, al uso de léxico especializado, a la posibilidad de utilización de formas verbales variadas, etc. De acuerdo con el objetivo de la investigación y teniendo en cuenta los diferentes niveles del texto, analizamos las recetas en shipibo y en castellano para ver si en ambas lenguas los alumnos muestran las mismas capacidades.

\subsection{Sujetos}

Los sujetos de la investigación son alumnos de $5^{\circ}$ y $6^{\circ}$ grado de educación primaria de una escuela situada en la comunidad nativa Puerto Firmeza del distrito de Yarinacocha, departamento de Ucayali, Perú. Son niños que tienen el shipibo como Lı y aprenden el castellano como L2 en la escuela. En la comunidad utilizan mayormente el shipibo como lengua familiar, aunque algunos de los alumnos utilizan tanto el castellano como el shipibo en el hogar. Dadas las características de la escolarización en esa zona, el docente debe atender a alumnos de distintas edades que a veces se integran en un mismo grado; por esa razón, en el aula donde se recogieron los textos solicitados había alumnos de entre 11 y 13 años. Para el estudio seleccionamos únicamente los alumnos que escribieron el texto en shipibo y en castellano, por lo que el grupo quedó formado por 13 alumnos de entre 11 y 13 años que cursaban $5^{\circ}$ y $6^{\circ}$ grado de primaria, es decir, quinto ciclo.

\subsection{Dispositivo y corpus. Criterios para el análisis de los textos}

Se proporciona a los alumnos una consigna para escribir una receta de cocina de un plato típico de su comunidad, pero con la particularidad de que los receptores de esa receta serían otros alumnos de primaria del País Vasco. Con la ayuda del profesor, eligen una receta y todos ellos la escriben un día en shipibo y otro en castellano. Así, obtenemos 13 recetas escritas en shipibo y 13 escritas en castellano por los mismos escolares. 
A fin de obtener una visión de las capacidades que muestran estos alumnos en la escritura del género textual que concierne a la investigación (las recetas de cocina), se comenzará dando cuenta de la longitud de los textos. A continuación, se abordará el análisis de estos en tres niveles de la arquitectura textual: infraestructura textual, mecanismos de conexión y cohesión, y mecanismos de responsabilidad enunciativa. Se integran en esos niveles algunas de las características más específicas de las recetas de cocina subrayadas por Adam (2001): estructura, organización temporal, léxico especializado, actualización de las acciones por medio de las formas verbales personales e impersonales.

\subsubsection{Infraestructura textual}

En el caso de las recetas de cocina, aun cuando admiten múltiples variaciones, cabe esperar una estructura canónica compuesta por estas partes: título, listado de ingredientes, descripción de los distintos pasos y, opcionalmente, infografía y recomendaciones o consejos (Adam, 2001: 33). No obstante, incluso cuando en el texto estén presentes todas esas fases, puede haber diferencias en el grado de desarrollo de cada una.

\subsubsection{Mecanismos de conexión}

Se abordan, en primer lugar, las características relativas a la organización temporal del proceso. Una de las características propias de las recetas de cocina es la imposición de una temporalidad o un orden lógico en la secuencia de elaboración, ya que su modificación puede alterar el proceso o incluso imposibilitar el resultado final; otra característica es que los organizadores temporales, además de precisar la sucesión, permiten precisar la duración de las operaciones y suboperaciones de las recetas. Se recuerda, no obstante, que esta secuenciación puede expresarse también con total ausencia de organizadores temporales. La secuenciación puede ser asumida en ese caso por marcas de puntuación o por elementos tipográficos de segmentación muy variados. Por eso, en segundo lugar, se examina la macrosegmentación tipográfica, que abarca la utilización en el texto de títulos, subtítulos, viñetas para las enumeraciones y los signos de puntuación, etc., elementos, en definitiva, que contribuyen a segmentar y organizar el contenido del texto.

\subsubsection{Mecanismos de cohesión nominal y verbal}

Entre los mecanismos de cohesión, se incluye el léxico que contribuye a la cohesión nominal del texto por medio de las retomas y de los procesos anafóricos. La receta de cocina conlleva el uso de un léxico nominal especializado (denominación de los alimentos, utensilios, proce- 
sos...), además de un léxico verbal acorde con la interacción sociodiscursiva de la que se trata. Por eso, siguiendo en el mismo nivel, se analiza también la cohesión verbal (formas y tiempos verbales, rupturas temporales...). Una de las peculiaridades de la receta es que las acciones temporales pueden ser expresadas con modos y tiempos verbales muy variados: imperativo, presente, futuro e incluso formas no personales como el infinitivo.

\subsubsection{Mecanismos de asunción de responsabilidad enunciativa}

Estos mecanismos contribuyen a establecer la coherencia prágmatica del texto, explicitando evaluaciones sobre su contenido temático (modalizaciones) y las fuentes de dichas evaluaciones (voces). Según señala Adam (2001), en las recetas las acciones pueden aparecer modalizadas por verbos como "deber" o "poder" que señalan de forma precisa la forma de hacer o llevar a cabo el proceso de elaboración. En principio, en un texto como la receta son previsibles proposiciones de valor ilocutivo fuerte como actos directivos marcados por el imperativo y algo menos por el infinitivo (Adam, 2001).

Respecto al uso de las voces, se analizan las del autor empírico del texto y las del destinatario de las recetas para indagar cuál es la característica enunciativa que presentan los textos de los alumnos.

\section{Resultados}

La mayoría de los textos presenta la estructura genérica de la receta de cocina, aunque ninguno de ellos hace uso de imágenes. A continuación, se detallan los resultados obtenidos.

\subsection{Longitud de los textos en número de palabras}

Si bien las diferentes propiedades de las lenguas pueden condicionar en cierta medida la longitud de los textos (T), contabilizamos el número de palabras totales del corpus de cada lengua y su promedio (tabla 1). Este valor no es muy significativo por sí mismo, aunque en el caso de las narraciones orales la longitud puede considerarse un criterio de desarrollo lingüístico (García-Azkoaga y otros, 2009). A grandes rasgos, este dato permite inferir en qué medida los alumnos han sido capaces de escribir la receta de cocina en las dos lenguas.

Las diferencias entre una y otra lengua pueden deberse a las características morfosintácticas de cada una, pues el shipibo es una lengua aglutinante y seguramente un mismo contenido puede expresarse con un menor número de palabras. En todo caso y pese a ejemplos de 


\section{TABLA 1}

Longitud de los textos

\begin{tabular}{lccccccccccccccc} 
TEXTOS & $\mathbf{T}$ & $\mathbf{T}$ & $\mathbf{T}$ & $\mathbf{T}$ & $\mathbf{T}$ & $\mathbf{T}$ & $\mathbf{T}$ & $\mathbf{T}$ & $\mathbf{T}$ & $\mathbf{T}$ & $\mathbf{T}$ & $\mathbf{T}$ & $\mathbf{T}$ & $\mathbf{N}^{0}$ TOTAL & PROMEDIO \\
& $\mathbf{1}$ & $\mathbf{2}$ & $\mathbf{3}$ & $\mathbf{4}$ & $\mathbf{5}$ & $\mathbf{6}$ & $\mathbf{7}$ & $\mathbf{8}$ & $\mathbf{9}$ & $\mathbf{1 0}$ & $\mathbf{1 1}$ & $\mathbf{1 2}$ & $\mathbf{1 3}$ & PALABRAS & \\
Shipibo & 42 & 67 & 46 & 65 & 43 & 69 & 30 & 25 & 43 & 60 & 27 & 55 & 29 & 601 & 46,23 \\
\hline Castellano & 84 & 68 & 52 & 67 & 72 & 91 & 56 & 30 & 55 & 51 & 45 & 50 & 38 & 759 & 58,38 \\
\hline
\end{tabular}

discordancia llamativa ( $T 1, T 5, T 7, T 11$, por ejemplo), hay un dato que destaca especialmente: el texto más largo en ambas lenguas pertenece al mismo estudiante; lo mismo sucede en caso del texto más corto.

\subsection{Infraestructura textual}

En la tabla 2 se aprecia cómo se ajustan las producciones de los alumnos a la estructura canónica de las recetas sugerida por Adam (2001).

\section{TABLA 2}

Estructura de las recetas

\begin{tabular}{|c|c|c|c|}
\hline & ESTRUCTURA & $\begin{array}{c}\text { SHIPIBO } \\
\text { L1 }\end{array}$ & $\begin{array}{c}\text { CASTELLANO } \\
\text { L2 }\end{array}$ \\
\hline \multirow{2}{*}{ Título } & Anticipa con precisión el contenido & 13 & 10 \\
\hline & Anticipa solo parcialmente el contenido & - & 1 \\
\hline \multicolumn{2}{|l|}{ Ingredientes } & 11 & 9 \\
\hline \multicolumn{2}{|l|}{ Imagen } & - & - \\
\hline \multirow{3}{*}{$\begin{array}{l}\text { Proceso de } \\
\text { elaboración }\end{array}$} & Se describen todos los pasos y en el orden adecuado & 4 & 6 \\
\hline & Se describen todos los pasos pero no en el orden adecuado & 0 & 1 \\
\hline & Faltan algunos pasos & 9 & 6 \\
\hline \multicolumn{2}{|c|}{ Recomendaciones } & 1 & 1 \\
\hline \multicolumn{2}{|c|}{ Existe una expresión de cierre para dar fin a la receta } & 0 & 5 \\
\hline
\end{tabular}

En el caso del shipibo, los trece textos Ilevan el mismo título (Piro kawa), lo cual permite adelantar adecuadamente la información sobre el contenido de la receta. Excepcionalmente, en uno de los casos aparece el subtítulo debajo del título (Rekenparira non biai - primero coge- 
mos-) que se refiere a los ingredientes. En cuanto a las recetas escritas en castellano, de los trece textos analizados falta el título en dos de ellos (Patarashca de piro) y en uno no aparece con precisión (Cawara - cahuara-), sino que se recurre a la denominación en castellano amazónico del nombre del pescado.

En lo concerniente a los ingredientes, estos aparecen explícitamente mencionados en 11 de los textos en shipibo y en 9 de los 13 escritos en castellano, si bien la forma de presentarlos difiere de unos textos a otros. La mayoría de las veces aparecen en forma de listado y al final del texto (8 en el caso del shipibo y 10 en el caso del castellano). Solo en 3 textos en shipibo y en 2 textos en castellano el listado aparece al comienzo. En el caso del castellano, hay un alumno que los integra en el texto en la fase de elaboración. Por otro lado, solo en 5 textos, y en castellano, los listados aparecen bajo un subtítulo (“ingredientes”, "condimentos”...). Por otro lado, no se especifican nunca las cantidades.

Es interesante mencionar que, al comparar los pares de textos producidos por los 13 alumnos, apreciamos que 9 tienen la misma estructura en ambas lenguas, mientras que 4 difieren, ya sea porque en uno se incluyen los ingredientes y en el otro no, o porque uno está titulado y el otro no. En lo que se refiere al proceso de elaboración, si bien está presente en todos los textos, no todos los alumnos expresan las acciones con el mismo grado de desarrollo; de ahí las tres gradaciones que aparecen en la tabla 2. Se han considerado los pasos básicos de la receta: destripar y lavar el pescado, preparar la hoja de plátano o de bijao, añadir los ingredientes, envolverlo y atarlo con una soga², ponerlo en la candela y, cuando está cocinado, comerlo.

De manera general, se observa que, cuando los alumnos tienen presentes todos los pasos del proceso en shipibo (T2, T4, T6, T12), lo hacen también en castellano, pero también se aprecia que es más habitual que falten algunos pasos en los textos escritos en shipibo ( $T_{1}, T_{3}, T_{5}$, T7, T8, T9, T10, T11, T13) que en castellano. Cabe pensar, por tanto, en una correlación con las capacidades escritas desarrolladas en una y otra lengua.

Por otra parte, además de las indicaciones indispensables para la elaboración del plato, la receta puede contener consejos o recomendaciones sobre la forma de potenciar algún sabor, hacerlo más sabroso, mejorar la presentación, conservarlo, etc. Solo en el T8, en shipibo, aparece la recomendación de que se puede comer con ají y limón; y en el Tıı, en castellano, aparece que "se le come con su plátano cocinado".

2 "Soga" es la palabra utilizada por el alumno para designar la cuerda fina o cordel con el que se ha de atar el pescado para su preparación. 
En lo que se refiere a la parte final de la receta, lo habitual en los textos analizados es que finalicen con el último paso del proceso: "después esperamos para comer"3 (Т3); "cuando está bueno cómalo" (T5); "cuando ya está cocinado ya vamos a comer" (T6); "y después lo asamos" (T13); "Watapekao non moa piai (después de cocinado lo comemos)" (T2); "Ja waketianra moa non piai (cuando ya está cocinado, listo, lo comemos)" (T3); "Japekao maton piai (después ustedes comen)" (T13). No obstante, en algunos casos (5 para el castellano), una vez finalizada la receta, aparecen fórmulas de cierre adicionales: "así se hace la patarashca (de piro)" (T1, T7); "eso es todo" (T3). En shipibo, no se han encontrado ese tipo de expresiones.

\subsection{Organización temporal del proceso de elaboración}

Los organizadores textuales constituyen cadenas de unidades lingüísticas de naturaleza muy diversa que contribuyen a la segmentación y articulación de las diferentes partes del texto. En el caso de las recetas son los organizadores temporales los que tienen un papel más relevante en la cohesión textual, pues organizan de forma ordenada la sucesión de los pasos que deben seguirse durante la elaboración para que el resultado sea el esperado. En la tabla 3 se observan cuáles son los más empleados en cada lengua.

\section{TABLA 3}

Organizadores temporales utilizados por los alumnos en cada lengua

\begin{tabular}{lclc} 
ORGANIZADOR TEMPORAL & $\begin{array}{c}\text { SHIPIBO } \\
\text { L1 }\end{array}$ & ORGANIZADOR TEMPORAL & $\begin{array}{c}\text { CASTELLANO } \\
\text { L2 }\end{array}$ \\
\hline Rekenpari(ra)(Primero) & 12 & Primero & 12 \\
\hline Axon(ra)(Después) & 12 & Después & 17 \\
\hline Pekao (Después) & 3 & Y después & 24 \\
\hline Japekao(ra) (Después) & 33 & Luego & 5 \\
\hline Jainxon(ra) (Después) & 10 & Yluego & 7 \\
\hline Jaskaxon (Después) & 5 & Y de ahí & 2 \\
\hline Jaska pekao(ra) (Luego) & 1 & Cuando & 8 \\
\hline Watapekao (Después de cocinado) & 1 & & 2 \\
\hline Waketian(ra) (Cuando está cocinado) & 2 & Después cuando & 2 \\
\hline
\end{tabular}

3 Presentamos los ejemplos tal como han sido escritos por los alumnos. 


\begin{tabular}{|c|c|c|c|}
\hline $\begin{array}{l}\text { Moa waketian(ra) (Ya } \\
\text { cuando está cocinado) }\end{array}$ & 2 & & \\
\hline Moa ikentian (Ya cuando está listo) & 2 & & \\
\hline $\begin{array}{l}\text { verbo+después (ejemplo: } \\
\{p o t e+x o n\} \text { 'después de destripar') }\end{array}$ & 14 & & \\
\hline Seneanxo (Hecho esto) & 3 & & \\
\hline Total & 100 & Total & 77 \\
\hline $\begin{array}{l}\text { \% respecto cantidad total de } \\
\text { palabras del conjunto de los textos }\end{array}$ & 16,63 & $\begin{array}{l}\text { \% respecto cantidad total de } \\
\text { palabras del conjunto de los textos }\end{array}$ & 10,14 \\
\hline
\end{tabular}

Los textos en shipibo presentan una mayor gama de conectores, lo cual podría explicarse por las propias características de las lenguas objeto de análisis, pero también por el hecho de que el shipibo es la lengua materna de los alumnos y, en consecuencia, poseen un repertorio más amplio.

En ambas lenguas la secuenciación temporal del proceso se realiza, principalmente, por organizadores temporales sencillos como "y", "después", "luego", "y luego"..., para el castellano, y numerosas formas equivalentes al castellano "después" en el caso del shipibo (axon(ra), pekao, japekao(ra), etc.). Todos esos elementos funcionan como meros encadenadores del discurso, pero no añaden ningún matiz especial sobre el mismo.

Existe otro grupo de organizadores temporales que se utilizan menos en ambas lenguas, pero que sirven para perfilar el proceso de elaboración. Se trata de los organizadores "cuando" y del doble organizador "cuando después", cuando van acompañados de una expresión que remite al paso anterior para darlo por finalizado antes de empezar con el siguiente. Por ejemplo, "cuando ya está listo”, "después cuando ya está (cocinado)", "cuando sus ingredientes ya están listos" (T4), "Moa waketian (ya cuando está cocinado)", "Moa ikentian (ya cuando está listo)", etc. También aparece la forma "y de ahí” en castellano. Excepcionalmente, aparece la expresión en shipibo "Seneanxo (Hecho esto)", que, sin embargo, no aparece en ninguno de los textos en castellano. Ese tipo de expresiones temporales, además de encadenar las acciones, también tienen el efecto de enfatizar y dar importancia a las acciones que se están desarrollando. Son expresiones que contribuyen a enriquecer el valor de los enunciados y ponen de manifiesto un mayor control sobre el discurso. Con su utilización no solo se unen dos enunciados, sino que se establece una relación entre ellos, de forma que se retoma el contenido del enunciado anterior y se marcan de alguna manera las condiciones en las que se puede seguir con la siguiente acción (“cuando está cocinado”, “cuando está listo”...). Este tipo de organizadores temporales, además de contribuir a conexionar el texto, también ejercen un efecto sobre la cohesión y la forma en que va progresando la información. 
Cabe constatar que, en lo referente al shipibo, existe la forma \{verbo+-xon\} que los shipibo-hablantes traducen al castellano como "después de + verbo". Por ejemplo, potexon (después de destripar), chokaxon (después de lavar). Eso es recurrente en varios textos. De hecho, en el análisis realizado por Valenzuela (2013), se determina que el morfema \{-xon\} es una marca de sujeto idéntico previo de un verbo transitivo, es decir, que el sujeto del enunciado es el mismo que el del enunciado anterior. En ningún caso aparecen estos últimos organizadores temporales junto con un \{verbo+-xon\}. En tal sentido, en el caso de la receta, este organizador sirve como cierre de la acción anterior indicando cuándo se puede proceder al paso siguiente:

(1) "Japekaora non chokai, chokaxonra non paranta pei biai [...]" (después lavamos, después de lavar, cogemos la hoja de plátano ...)(T3).

(2) "Rekenpari non piro poteai, potexon non chokai [...]" (primero destripamos la cahuara, después de destripar, lo lavamos) (T4).

\subsection{La macrosegmentación tipográfica}

A continuación, se analiza la utilización de subtítulos, viñetas para segmentar el texto y signos de puntuación. No nos detenemos en el título porque se abordó previamente como parte esencial de la infraestructura textual. En shipibo, 3 alumnos utilizan un subtítulo para los ingredientes. La iniciativa de un alumno es formar una oración para hacer la referencia rekenparira non biai, que significa 'primero cogemos'; los otros dos alumnos repiten el título de la receta y lo escriben antes de los ingredientes: piro kawa, que significa 'patarashca de cahuara'. En castellano, sin embargo, algunos alumnos utilizan un subtítulo para los ingredientes ("Ios ingredientes" en tres textos, "condimento del piro" en dos) y uno lo hace para el proceso de elaboración ("para preparar"). Las marcas tipográficas especiales como guiones (-) se utilizan en el listado de los ingredientes en 6 de los textos escritos en shipibo y en 3 textos en castellano; en esta lengua se utiliza la numeración en uno de los textos.

Respecto a los signos de puntuación, se aprecia una ausencia generalizada de su uso en las dos lenguas.

\subsection{Cohesión nominal}

Tanto en shipibo como en castellano se emplea un léxico propio de las recetas culinarias. No obstante, se trata de léxico característico de su contexto sociocultural, por lo que la repetición léxica y la falta de retomas explicativas pueden dificultar en ocasiones la comprensión del contenido y hacerlo poco accesible a quien no comparte las coordenadas lingüístico- 
-culturales (cfr. Garcia-Azkoaga, en prensa). A través de la mención de los ingredientes o de los utensilios de cocina se pueden ir tejiendo a lo largo del texto relaciones anafóricas que permiten la retoma de esos elementos a lo largo del texto, pero en los textos analizados esas relaciones anafóricas raramente aparecen y, cuando lo hacen, se establecen básicamente por medio de la reiteración léxica.

(3) “Primero destripa y lava el piro después de lavar corta hoja de plátano y luego corta el piro $[\ldots] "(T 5)$.

(4) "Rekenpari non piro poteai ja potexon non chokai [...] jaskaxon non piro tashi rasai [...]" (primero destripamos la cahuara, eso después de destripar, lavamos [...] después salpicamos sal a la cahuara [...]) (T6).

Por otro lado, las repeticiones se evitan básicamente mediante la utilización de los pronombres "le” y "lo", que aportan cohesión nominal al establecer una relación anafórica. Son formas abundantes en castellano, aunque se observa que su utilización no siempre es la adecuada ("primero se lo destripa", "lo destripamos el piro", "vamos lo a...", "le lavamos", "lo echamos cebolla”). En cuanto a los textos en shipibo, la mayoría de alumnos (8 textos) utiliza una concatenación de oraciones simples en las cuales no se repite el léxico, pero tampoco se usan anáforas. No obstante, algunos alumnos (3 textos), cuando realizan una retoma, emplean el demostrativo ja, que significa 'ese', 'eso', si el elemento al que se hace referencia está próximo. Si el referente se encuentra más alejado - por ejemplo, en una oración posterior, como en (4)-, se prefiere una repetición léxica. Otros alumnos en cambio, solo utilizan repeticiones. Así sucede en otros 2 textos.

\subsection{Cohesión verbal}

Líneas arriba se mencionó que una de las características de las recetas, en lo que concierne a los tiempos verbales, es la posibilidad de utilizar formas muy variadas, por lo que, seguidamente, se analizarán cuáles son las formas más habituales en cada lengua y si se producen o no rupturas temporales por un uso o una combinación inadecuada de esas formas temporales.

La mayoría de los textos (6 en castellano y 11 en shipibo) están escritos en presente y en primera persona del plural. No obstante, un texto en shipibo (T13) cambia la redacción en primera persona del plural (non) por la segunda persona del plural en sus dos últimas oraciones (maton). En lo que respecta al castellano, en uno de los textos el uso del presente adquiere un sentido de futuro por efecto del organizador temporal que le precede: "después vamos a cortar" (T13). Además de los textos en presente, hay cuatro que están escritos en forma impersonal. También se encuentran otras formas temporales o incluso combinación 
de varias formas - no siempre acertadas - En este sentido, en uno de los textos redactados en imperativo (T8), se hace uso del presente en la última parte del proceso ("Iava su carne [...] después como"), lo que produce un efecto de ruptura temporal. Se da la circunstancia de que este texto, en particular, está menos elaborado que el resto y pertenece al alumno que ha escrito los textos más cortos en ambas lenguas. Dicha ruptura temporal no es, sin embargo, tan relevante cuando en un mismo texto aparecen las formas de presente en combinación con las de futuro, como sucede en T10: "primero lo (que) asemos es cortar el piro y luego sacaremos su tripa...". Lo mismo podemos decir cuando se trata de la aparición combinada de la forma personal con la forma impersonal, como sucede en T1: "Cuando hacemos [...] primero destripamos [...] se desatiza la candela [...] se come”.

En el caso del shipibo, solo en dos textos se utiliza el presente combinado con el infinitivo. Sin embargo, en ambos casos el uso del infinitivo es considerado un error gramatical, pues el pronombre de primera persona del plural antecede al verbo en infinitivo, cuya marca es \{-ti\}, en lugar de la aparición canónica del verbo junto a la marca \{-ai\}:

(5) "jaskaxon nonxoimati” 'después nosotros asar' (T7) (correcto: jaskaxon non xoimai 'después asamos').

(6) “non chokati” ‘nosotros destripar' (T11) (correcto: non chokai ‘destripamos’).

También se constata que en ambas lenguas los alumnos optan por construcciones verbales que se corresponden con las particularidades del género textual solicitado, principalmente formas de presente. Merece, asimismo, enfatizarse que la forma impersonal no es muy habitual en castellano, mientras que en shipibo no se usa. Por otro lado, de manera general, la ruptura temporal que se aprecia en algunos textos en castellano parece responder a la dificultad percibida por los alumnos a la hora de gestionar los tiempos verbales en esa L2, un aspecto que requeriría un análisis más profundo.

\subsection{Mecanismos de asunción de responsabilidad enunciativa}

Si bien en las recetas cabría esperar el uso de verbos deónticos como "poder" o "deber", solo en un texto en castellano se utiliza el verbo "deber" (con un error ortográfico): "devemos (sic) traer una parrilla" (T6). En otros dos textos la modalización se ejerce por medio del uso del imperativo: "Primero destripalo y lava el piro" (T5), "después laba (sic) su carne” (T8) (con un error ortográfico). En shipibo se observa el uso de expresiones como Akin, traducido al castellano como "hazlo" (T4, T6), y que denotan un acto directivo de valor ilocutivo similar al imperativo del castellano. Más allá de los ejemplos citados, no se encuentran ni en shipibo ni en castellano adjetivos o expresiones adverbiales que tengan una función modalizadora en el discurso. 
Respecto al uso de las diferentes voces, salvo en tres textos castellanos, escritos en forma impersonal, se prefiere la primera persona del plural y no hay ninguna marca referencial o deíctica que remita a un posible interlocutor. No obstante, tal y como se mencionó anteriormente, sí hay un estudiante que cambia durante su redacción en shipibo de la primera a la segunda persona del plural.

\section{Conclusiones}

Recuérdese, en primer lugar, que el objetivo de este trabajo no es mostrar las capacidades que los alumnos bilingües tienen en castellano, sino indagar en el potencial de una educación intercultural bilingüe para contribuir al desarrollo de la competencia comunicativa en las dos lenguas (shipibo y castellano) que se utilizan en la escuela. Así pues, en las páginas precedentes se ha puesto de manifiesto que, en general, aun cuando no ha habido una enseñanza explícita sobre el género textual como tal, los alumnos son capaces de producir, tanto en shipibo como en castellano, textos fácilmente identificables como recetas de cocina. Todos presentan la estructura textual básica y las marcas de coherencia, cohesión y modalización propias de este género, si bien es cierto que los textos son, en general, poco elaborados y existen considerables errores morfosintácticos.

Se podría afirmar que todos los alumnos tienen un conocimiento general sobre el funcionamiento de la lengua que les permite poner en práctica las habilidades lingüísticas y los recursos necesarios para producir el texto requerido, lo que corrobora, una vez más, la importancia de las prácticas sociodiscursivas que guían el uso de la actividad verbal en el seno de una comunidad. En cuanto a las diferencias entre el grado de elaboración de los textos en una u otra lengua, estas no son muy significativas. A la luz de los datos de que se dispone, tampoco se puede precisar cuál es la influencia y el impacto que ha tenido y tiene la EIB en la adquisición de las destrezas escritas en los alumnos, dado que no se puede conocer con certeza el peso que tiene cada lengua en el proceso de enseñanza-aprendizaje. Por lo tanto, en un futuro cercano sería deseable emprender un estudio más específico en el que se pudieran evaluar las capacidades de alumnos que tienen como lengua vehicular de la escuela solo el shipibo y alumnos que tienen como lengua vehicular solo el español.

Asimismo, resultaría también interesante comprobar los resultados de los aprendizajes tras la implementación de una secuencia didáctica específica para trabajar el género textual "receta de cocina". A este respecto, se comprueba el hecho de que el aprendizaje de la escritura se aborda como una tarea de transcripción de la lengua oral que se fundamenta principalmente en la corrección gramatical, en el seguimiento de la norma prescrita. En efecto, los textos producidos se adaptan al género textual requerido, pero discursiva y enunciativamente no presentan marcas que indiquen que la receta va dirigida a lectores que deben seguir las instrucciones para 
preparar la receta ellos mismos. Se constata, pues, una marcada ausencia de reflexión sobre el receptor, una circunstancia que está muy ligada a la forma en que se concibe la lengua durante el proceso de enseñanza-aprendizaje. Por consiguiente, pensamos que una didáctica basada en los géneros textuales podría contribuir notablemente a mejorar las estrategias discursivas y al desarrollo integral de las capacidades comunicativas en las dos lenguas. En ese sentido, y para contribuir a la mejora de los programas de educación intercultural bilingüe, se podrían abordar dos aspectos fundamentales en la didáctica de la lengua: a) refuerzo del trabajo escrito sobre los géneros textuales como objeto de aprendizaje para poder abordar los distintos niveles de la arquitectura textual en las dos lenguas y de forma coordinada, de manera que los aprendizajes de una sean aprovechados en la otra; b) formación y actualización lingüística de los docentes en ambas lenguas y en el uso de metodologías y recursos didácticos enfocados al aprendizaje de los géneros textuales, en definitiva, al uso social de la lengua.

Esta metodología de análisis permite abordar los aspectos comunicativos de la lengua y permite observar aspectos textuales obviados en los análisis gramaticales tradicionales. Paralelamente, además de aportar un nuevo enfoque didáctico, esta aproximación metodológica permite sentar las bases de un análisis más pormenorizado sobre las relaciones interlingüísticas y las trasferencias entre ambas, shipibo y castellano, en cada uno de los sujetos. Creemos que este trabajo, aún en etapa exploratoria, constituye una importante contribución al desarrollo de los estudios sobre el bilingüismo con lenguas originarias, especialmente en el caso de aquellas que son minoritarias.

\section{Bibliografía citada}

ADAm, Jean-Michel, 2001: “Entre conseil et consigne: les genres de l'incitation à l'action”, Pratiques $111 / 112,7-38$.

Amorrortu, Estíbaliz, y otros, 2006: Palabras y Mundos, Barcelona: Icaria Editorial.

Bronckart, Jean-Paul, 1996: Activité Langagière, textes et discourses, Lausana: Delachaux et Niestlé. [Traducción al castellano: Actividad verbal, textos y discursos, Madrid: Infancia y Aprendizaje, 2004].

Calvo Pérez, Julio, 2001: “Tipologia textual” en Ángel López y Manuel Prunyorosa (eds.): Fonaments de la comunicació, València: Universitat de València, 235-265.

De Pietro, Jean-François, y Bernard Schneuwly, 2003: "Le modèle didactique du genre: un concept de l'ingénierie didactique”, Les Cahiers Théodile 3, 27-52.

Díaz de Gereñu, Leire, y Ma Inés Garcia-Azkoaga, 2016: "Aprendiendo dos lenguas nuevas. Los cuentos orales de los niños inmigrantes”, Lengua y Migración /Language and Migration 8(1), 29-49. 
Dolz, Joaquim, y Roxane GaGnon, 2010: "El género textual, una herramienta didáctica para desarrollar el lenguaje oral y escrito”, Lenguaje 38 (2), 498-527.

Dolz, Joaquim, Michèle NoverRaz y Bernard Schneuwly, 2001: Séquences didactiques pour l'oralet pour l'écrit. Notes méthodologiques, vol. II, Bruxelles: De Boeck.

GarcíA-Azkoaga, Inés M. a, en prensa: "La receta de cocina, un recurso multidimensional para la didáctica de la escritura”, comunicación presentada en el VIII Congreso Internacional de la Cátedra Unesco de Lectura y Escritura.

García-Azkoaga, Inés Ma e e Itziar IdiazÁbal (eds.), 2015: Para una ingeniería didáctica del plurilingüismo, Bilbao: Servicio Editorial de la Universidad del País Vasco / Euskal Herriko Unibertsitatea.

García-Azkoaga, Inés $M^{a}$, Itziar Idiazábal y Luis Ma LaRRingan, 2009: "Contar el mismo cuento a los 5 y a los 8 años. Una explicación desde la perspectiva del interaccionismo sociodiscursivo", Revista de Estudos Linguísticos / Linguistic Studies 3, 211-226.

IdiAzÁBAL, Itziar, e Inés Ma García-Azkoaga, 2015: "Castellano L1 y L2 en escolares bilingües vascocastellanos. La escritura de recetas de cocina” en Dora Riestra, Stella Maris Tapia y Ma Victoria Goicoechea Gaona (eds.): Cuartas Jornadas Internacionales de Investigación y Prácticas en Didáctica de las lenguas y las literaturas, Bariloche, Argentina, 203-221 [disponible en: http:// editorial.unrn.edu.ar/components/com_booklibrary/ebooks/Congresos_Jornadas_Didactica_Lenguas_Literaturas_1, fecha de consulta 03-07-2016].

MANTERola, Ibon, 2012: Euskerazko murgilketa haur hezkuntzan: euskera-gaztelania elebitasunaren azterketa. Ahozko ipuinen analisi diskurtsiboa eta didaktikoa. Tesis de doctorado, Universidad del País Vasco (UPV/EHU).

Valenzuela, Pilar M., 2001: "Características Morfosintácticas del Idioma Shipibo-Konibo del Ucayali” en Bob De Jonge (ed.): Actas del I Congreso de la Asociación de Lingüística y Filología de América Latina (ALFAL), vol. 13 [disponible en: http://elies.rediris.es/elies13, fecha de consulta 27-11-2015].

Valenzuela, Pilar M., 2006: "Los verbos del castellano en el shipibo-konibo: Aportes a un propuesto universal del contacto lingüístico" en Claudine ChAmoReAu y Yolanda LASTRA (eds.): Dinámica Lingüística de las Lenguas en Contacto, Hermosillo, México: Universidad de Sonora, 141-168.

Valenzuela, Pilar M., 2013: "El sistema de concordancia del participante en las lenguas pano y sus implicancias para el conocimiento de la protolengua”, Revista Brasileira de Linguística Antropológica 5(1), 117-157.

ZARIQUIEY, Roberto, 2006: "Reinterpretación fonológica de los préstamos léxicos de base hispana en la lengua shipibo-conibo", Boletín de la Academia Peruana de la Lengua 41, 59-78. 